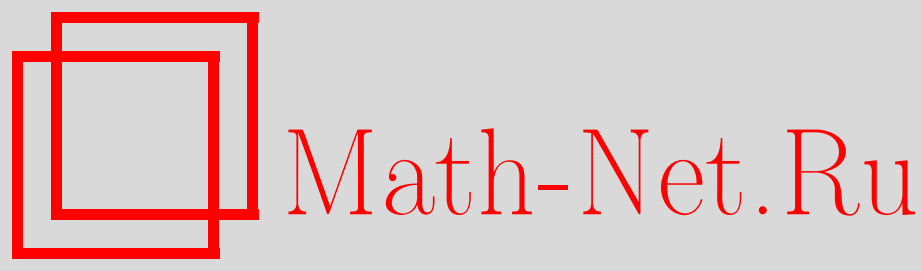

Е. Юрчишинова, М. Юрчишин, Р. Ремецки, Влияние спиральности на турбулентное число Прандтля: двухпетлевое приближение, ТМФ, 2011, том 169, номер 2, 241-252

DOI: https://doi.org/10.4213/tmf6725

Использование Общероссийского математического портала Math-Net.Ru подразумевает, что вы прочитали и согласны с пользовательским соглашением http://www.mathnet.ru/rus/agreement

Параметры загрузки:

IP : 3.80 .181 .102

26 апреля 2023 г., 03:12:22

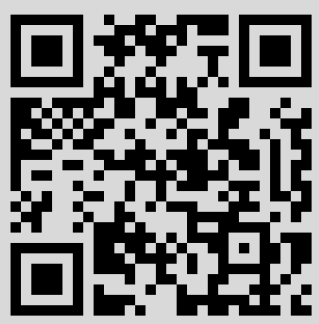




\title{
ФИЗИКА
}

Том 169, № 2

ноябрь, 2011

(C) 2011 г. . Юрчишинова*, М. Юрчишин*, Р. Ремецки*

\section{ВЛИЯНИЕ СПИРАЛЬНОСТИ НА ТУРБУЛЕНТНОЕ ЧИСЛО ПРАНДТЛЯ: ДВУХПЕТЛЕВОЕ ПРИБЛИЖЕНИЕ}

\begin{abstract}
С использованиием метода теоретико-полевой ренормализационной группы в рамках двухпетлевого приближения изучается влияние спиральности (нарушения пространственной четности) на турбулентное число Прандтля в модели скалярного поля, пассивно увлекаемого спиральной турбулентной средой, которая задается стохастическим уравнением Навье-Стокса с самоподобной гауссовой случайной перемешивающей силой, $\delta$-коррелированной по времени с коррелятором, пропорциональным $k^{4-d-2 \varepsilon}$. Кратко рассматривается влияние спиральности на устойчивость соответствующего скейлингового режима. Показано, что наличие спиральности увеличивает значение турбулентного числа Прандтля вплоть до 50\%-го превышения над неспиральным значением.
\end{abstract}

Ключевые слова: развитая турбулентность, пассивная адвекция, спиральность, ренормализационная группа.

\section{1. ВВЕДЕНИЕ}

Одной из характеристик диффузионных процессов в жидкостях и газах является безразмерное отношение коэффициента кинематической вязкости к коэффициенту тепловой диффузивности (в задаче о температурной диффузии), а также отношение коэффициента кинематической вязкости к молекулярной диффузивности (в задаче о концентрации примеси). Первое отношение известно как число Прандтля, а второе - как так называемое число Шмидта. Когда жидкость или газ находятся в состоянии полностью развитой турбулентности, процессы диффузии быстро ускоряются, что выражается в появлении эффективного значения коэффициентов диффузии, а именно турбулентной тепловой диффузивности и турбулентной молекулярной диффузивности. Отношение турбулентной вязкости к турбулентной тепловой диффузивности есть так называемое турбулентное число Прандтля $\mathrm{Pr}_{\mathrm{t}}$, которое представляет собой универсальную величину, т. е. не зависит от свойств конкретной жидкости или газа. Экспериментально полученные значения этого числа лежат в интервале 0.7-0.9 [1], [2].

*Institute of Experimental Physics, Slovak Academy of Sciences, Košice, Slovakia. E-mail: remecky@saske.sk 
Хорошо известно, что эффективным подходом к исследованию универсальных свойств процессов в среде с полностью развитой турбулентностью является метод ренормализационной группы (РГ) [3]-[6]. Метод РГ можно также использовать для вычисления турбулентного числа Прандтля, которое задается в виде ряда теории возмущений по соответствующему параметру разложения $\varepsilon$ модели (см. раздел 2 настоящей работы). Совсем недавно оно было вычислено во втором порядке теории возмущений с использованием теоретико-полевого метода РГ [7], [8]. Показано, что двухпетлевая поправка к турбулентному числу Прандтля оказывается неожиданно очень малой, меньше $2 \%$ от главного однопетлевого вклада $\operatorname{Pr}_{\mathrm{t}}^{(1)}=0.7179$ [9]. Это означает, что турбулентное число Прандтля очень устойчиво по отношению к разложению по теории возмущений. С другой стороны, полученное двухпетлевое значение турбулентного числа Прандтля $\mathrm{Pr}_{\mathrm{t}}=0.7051$ [8] находится также в хорошем согласии с экспериментальными значениями [1], [2].

Тем не менее до настоящего времени остается открытым целый ряд вопросов. В первую очередь это вопрос о влиянии разнообразных нарушений симметрии на процессы турбулентности глубоко внутри так называемого инерциального интервала $l \ll r \ll L$, т. е. вдали от внешнего (интегрального) масштаба $L$, на котором поступает энергия для поддержания системы в стационарном состоянии, и в то же время вдали от внутреннего (вязкого) масштаба $l$, где энергия диссипирует. Этот вопрос представляет интерес, поскольку экспериментальный факт состоит в том, что процессы гомогенизации начальных и граничных условий (накачка энергии при больших масштабах), приводящие к развитой турбулентности, являются намного более медленными, чем предполагалось ранее (см., например, работы [10] и приведенную в них библиографию).

В этой связи в настоящей работе мы исследуем процессы адвекции пассивного скалярного поля (например, поля температуры) турбулентной средой, задаваемой стохастическим уравнением Навье-Стокса со спиральной вынуждающей случайной силой, т. е. со случайной силой, нарушающей пространственную четность (с зеркальной асимметрией). Цель статьи - найти явную зависимость турбулентного числа Прандтля от параметра, который управляет наличием спиральности в турбулентной системе. Мы постараемся показать, что наличие спиральности может довольно сильно влиять на процессы диффузии в полностью развитой турбулентности, что согласуется с более ранними результатами, полученными в работе [11], где было показано, что возможно сильное влияние спиральности на коэффициент диффузии.

\section{2. МОДЕЛЬ}

Адвекция пассивного скалярного поля $\theta(x) \equiv \theta(t, \mathbf{x})$ (температуры, концентрации примесей и т. д.) несжимаемым полем скоростей Навье-Стокса $\mathbf{v}(x) \equiv \mathbf{v}(t, \mathbf{x})$ (где $\left.\partial_{i} v_{i}=0\right)$ описывается системой стохастических уравнений

$$
\begin{aligned}
\partial_{t} \theta+v_{i} \partial_{i} \theta & =\nu_{0} u_{0} \Delta \theta+f^{\theta}, \\
\partial_{t} \mathbf{v}+v_{i} \partial_{i} \mathbf{v} & =\nu_{0} \Delta \mathbf{v}-\partial_{i} P+\mathbf{f}^{\mathbf{v}},
\end{aligned}
$$

где уравнение (1) представляет собой уравнение адвекции-диффузии для скалярного поля, а уравнение (2) является стохастическим уравнением Навье-Стокса для 
поперечного (вследствие несжимаемости) поля скоростей. В уравнениях (1) и (2) используются стандартные обозначения $\partial_{t} \equiv \partial / \partial t, \partial_{i} \equiv \partial / \partial x_{i}, \Delta \equiv \partial^{2}$ - оператор Лапласа, $\nu_{0}$ - кинематическая вязкость (далее нижний индекс 0 обозначает затравочные параметры неперенормированной теории), $\nu_{0} u_{0}-$ коэффициент тепловой диффузивности (или молекулярной диффузивности), причем безразмерное обратное число Прандтля $u_{0}$ получается явно (в случае молекулярной диффузии оно известно как обратное число Шмидта), $P(x) \equiv P(t, \mathbf{x})$ - давление. Также в $(1),(2)$ и далее подразумевается суммирование по немым индексам. Случайный шум $f^{\theta}=f^{\theta}(x)$ выбран гауссовым с коррелятором

$$
D^{\theta}\left(x ; x^{\prime}\right)=\left\langle f^{\theta}(x) f^{\theta}\left(x^{\prime}\right)\right\rangle=\delta\left(t-t^{\prime}\right) C\left(\frac{\mathbf{r}}{L}\right), \quad \mathbf{r}=\mathbf{x}-\mathbf{x}^{\prime} .
$$

Здесь и далее от несущественной функции $C(\mathbf{r} / L)$ требуется только достаточно быстрое убывание при $r \equiv|\mathbf{r}| \gg L$ для некоторого интегрального масштаба $L$. Основная роль шума (3) состоит в поддержании стационарного состояния системы. C другой стороны, важным является явный вид поперечной случайной силы $\mathbf{f}^{\mathbf{v}}$, отнесенной к единичной массе. Обычно предполагается, что она также подчиняется гауссовому распределению с нулевым средним и коррелятором

$$
D_{i j}^{v}\left(x ; x^{\prime}\right)=\left\langle f_{i}^{\mathbf{v}}(x) f_{j}^{\mathbf{v}}\left(x^{\prime}\right)\right\rangle=\delta\left(t-t^{\prime}\right) \int \frac{d^{d} \mathbf{k}}{(2 \pi)^{d}} R_{i j}(\mathbf{k}) d_{f}(k) e^{i \mathbf{k} \cdot\left(\mathbf{x}-\mathbf{x}^{\prime}\right)},
$$

где $d$ - размерность $\mathbf{x}$-пространства, а $R_{i j}(\mathbf{k})$ - поперечный проектор. Таким образом описываются геометрические свойства случайной силы, в простейшем изотропном случае $R_{i j}(\mathbf{k})=\delta_{i j}-k_{i} k_{j} / k^{2}$. Функция энергии накачки $d_{f}(k)$ выбирается так, чтобы реализовать реалистичное, т. е. инфракрасное (ИК) поступление (посредством крупномасштабных водоворотов) энергии в систему, в то же время важно, чтобы функция $d_{f}(k)$ при больших $k$ имела степенную асимптотику. Последнее условие необходимо для применения теоретико-полевого метода РГ. Обоим условиям удовлетворяет функция $d_{f}(k)=D_{0} k^{4-d-2 \varepsilon}$ с положительной амплитудой $D_{0}>0$ и показателем $0<\varepsilon \leqslant 2[4]-[6]$. Наиболее реалистичным значением является $\varepsilon=2$ (см. монографии [5], [6]). В уравнении (4) требуемая ИК-регуляризация получается путем ограничения интегрирования снизу: $k \geqslant m$, где $m$ соответствует еще одному интегральному масштабу. Будем предполагать, что $L \gg 1 / m$. Для большего удобства полезно также ввести новую затравочную константу взаимодействия $g_{0}$ вместо $D_{0}$, определив ее как $D_{0} \equiv g_{0} \nu_{0}^{3}$. Кроме того, $g_{0}$ формально является малым параметром обычной теории возмущений и связана с характеристическим ультрафиолетовым (УФ) масштабом импульсов $\Lambda$ (или внутренней длиной $l \sim \Lambda^{-1}$ ) соотношением $g_{0} \simeq \Lambda^{2 \varepsilon}$. Галилеева инвариантность стохастической модели $(1)-(4)$ гарантируется временной декорреляцией случайных сил (3) и (4).

Переход к спиральной жидкости или газу соответствует отказу от сохранения пространственной четности, технически это выражается в том, что корреляционная функция задается в виде смеси тензора и псевдотензора. В нашем изотропном случае это означает, что поперечный проектор $R_{i j}(\mathbf{k})$ в уравнении (4) можно разбить на две части,

$$
R_{i j}(\mathbf{k})=P_{i j}(\mathbf{k})+H_{i j}(\mathbf{k})=\delta_{i j}-\frac{k_{i} k_{j}}{k^{2}}+i \rho \varepsilon_{i j l} \frac{k_{l}}{k},
$$


состоящие из неспирального стандартного поперечного проектора $P_{i j}(\mathbf{k})=\delta_{i j}-$ $k_{i} k_{j} / k^{2}$ и тензора $H_{i j}(\mathbf{k})=i \rho \varepsilon_{i j l} k_{l} / k$, который выражает наличие спиральности в потоке. Здесь $\varepsilon_{i j l}$ - полностью антисимметричный тензор Леви-Чивита ранга 3, а вещественный параметр спиральности $\rho$ характеризует величину спиральности. Вследствие требования положительной определенности корреляционнй функции абсолютное значение $\rho$ должно лежать в интервале $|\rho| \in[0,1]$. Физически это означает, что ненулевая спиральная часть отвечает за существование ненулевых корреляций $\langle\mathbf{v} \cdot \operatorname{rot} \mathbf{v}\rangle$ в системе.

\section{3. ТЕОРЕТИКО-ПОЛЕВАЯ ФОРМУЛИРОВКА МОДЕЛИ}

Используя общую теорему [12], можно переписать стохастическую модель (1)-(4) в виде эквивалентной теоретико-полевой модели для набора четырех полей $\Phi=$ $\left\{\mathbf{v}, \theta, \mathbf{v}^{\prime}, \theta^{\prime}\right\}$ с функционалом действия

$$
\begin{aligned}
S(\Phi)= & \frac{1}{2} \int d t_{1} d^{d} \mathbf{x}_{1} d t_{2} d^{d} \mathbf{x}_{2}\left[v_{i}^{\prime}\left(t_{1}, \mathbf{x}_{1}\right) D_{i j}^{v}\left(t_{1}, \mathbf{x}_{1} ; t_{2}, \mathbf{x}_{2}\right) v_{j}^{\prime}\left(t_{2}, \mathbf{x}_{2}\right)+\right. \\
& \left.+\theta^{\prime}\left(t_{1}, \mathbf{x}_{1}\right) D^{\theta}\left(t_{1}, \mathbf{x}_{1} ; t_{2}, \mathbf{x}_{2}\right) \theta^{\prime}\left(t_{2}, \mathbf{x}_{2}\right)\right]+ \\
& +\int d t d^{d} \mathbf{x}\left\{\theta^{\prime}\left[-\partial_{t}-\mathbf{v} \cdot \boldsymbol{\partial}+\nu_{0} u_{0} \Delta\right] \theta+\mathbf{v}^{\prime}\left[-\partial_{t}-\mathbf{v} \cdot \boldsymbol{\partial}+\nu_{0} \Delta\right] \mathbf{v}\right\},
\end{aligned}
$$

где $\mathbf{v}^{\prime}(x)$ и $\theta^{\prime}(x)$ - необходимые вспомогательные поля с теми же тензорными свойствами, что и у полей $\mathbf{v}(x)$ и $\theta(x)$, а $D^{\theta}$ и $D^{v}-$ корреляционные функции, заданные в (3) и (4) для случайных сил $f^{\theta}$ и $\mathbf{f}^{\mathbf{v}}$ соответственно (напоминаем, что предполагается необходимое суммирование по немым индексам).

Поскольку вспомогательное векторное поле $\mathbf{v}^{\prime}(x)$ также является поперечным, $\partial_{i} v_{i}^{\prime}=0$, в функционале (5) можно отбросить член, связанный с давлением, который имеет вид $\int d t d^{d} \mathbf{x} v_{i}^{\prime} \partial_{i} P$. После интегрирования по частям становится очевидно, что он обращается в ноль:

$$
\int d t d^{d} \mathbf{x} v_{i}^{\prime} \partial_{i} P=-\int d t d^{d} \mathbf{x} P \partial_{i} v_{i}^{\prime}=0 .
$$

Модель (5) соответствует стандартной теории возмущений, описываемой фейнмановскими диаграммами со следующими ненулевыми затравочными пропагаторами (в частотно-импульсном представлении):

$$
\begin{aligned}
\left\langle v_{i} v_{j}^{\prime}\right\rangle_{0} & =\left\langle v_{i}^{\prime} v_{j}\right\rangle_{0}^{*}=\frac{P_{i j}(\mathbf{k})}{-i \omega+\nu_{0} k^{2}}, & \left\langle v_{i} v_{j}\right\rangle_{0} & =\frac{g_{0} \nu_{0}^{3} k^{4-d-2 \varepsilon} R_{i j}(\mathbf{k})}{\left(-i \omega+\nu_{0} k^{2}\right)\left(i \omega+\nu_{0} k^{2}\right)}, \\
\left\langle\theta \theta^{\prime}\right\rangle_{0} & =\left\langle\theta^{\prime} \theta\right\rangle_{0}^{*}=\frac{1}{-i \omega+\nu_{0} u_{0} k^{2}}, & \langle\theta \theta\rangle_{0} & =\frac{C(\mathbf{k})}{\left(-i \omega+\nu_{0} u_{0} k^{2}\right)\left(i \omega+\nu_{0} u_{0} k^{2}\right)},
\end{aligned}
$$

где $C(\mathbf{k})$ - преобразование Фурье функции $C(\mathbf{r} / L)$ в уравнении (3). С другой стороны, вершины взаимодействия имеют вид

$$
-v_{i}^{\prime}\left(v_{j} \partial_{j}\right) v_{i}=\frac{v_{i}^{\prime} W_{i j l} v_{j} v_{l}}{2}, \quad \theta^{\prime}\left(v_{j} \partial_{j}\right) \theta=\theta^{\prime} V_{j} v_{j} \theta
$$

где (в частотно-импульсном представлении)

$$
W_{i j l}=i\left(k_{j} \delta_{i l}+k_{l} \delta_{i j}\right), \quad V_{j}=i k_{j}
$$




$$
\begin{aligned}
& \langle\theta \theta\rangle_{0}=\square \quad\left\langle v_{i} v_{j}\right\rangle_{0}=\ldots \ldots \ldots \\
& \left\langle\theta^{\prime} \theta\right\rangle_{0}=+\quad\left\langle v_{i}^{\prime} v_{j}\right\rangle_{0}=-1 \ldots \ldots \ldots
\end{aligned}
$$

Рис. 1. Графическое представление пропагаторов модели.

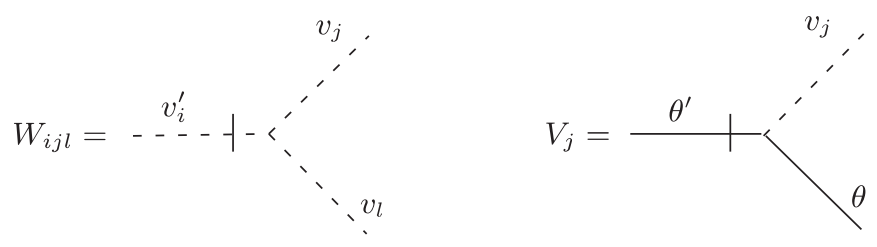

Рис. 2. Графическое представление вершин взаимодействия модели.

импульс $\mathbf{k}$ втекает в вершины через вспомогательные поля $\mathbf{v}^{\prime}$ и $\theta^{\prime}$ соответственно. Соответствующие графические представления ненулевых пропагаторов и вершин показаны на рис. 1 и 2.

Преимущество формулировки стохастической задачи, задаваемой уравнениями (1)-(4), через функционал действия (5), заключается в том, что она позволяет применять для анализа задачи корректно определенные теоретико-полевые способы действий, например метод РГ, причем статистические средние случайных величин в стохастической задаче заменяются на соответствующие функциональные средние с весом $e^{S(\Phi)}[4]-[6]$.

\section{4. РЕНОРМГРУППОВОЙ АНАЛИЗ}

Ренормгрупповой анализ основан на анализе УФ-расходимостей модели, которые определяются каноническими размерностями. Динамическая модель (5) относится к классу так называемых двумасштабных моделей [4]-[6], для которых каноническая размерность некоторой величины $Q$ задается двумя числами: импульсной размерностью $d_{Q}^{k}$ и частототной размерностью $d_{Q}^{\omega}$. Таким образом, размерность всех величин можно найти из требования, чтобы каждый член функционала действия был безразмерным по импульсу и по частоте, а также должны выполняться стандартные определения (условия нормировки) $d_{k}^{k}=-d_{x}^{k}=d_{\omega}^{\omega}=-d_{t}^{\omega}=1$ и $d_{k}^{\omega}=d_{\omega}^{k}=0$. Тогда полная каноническая размерность $d_{Q}$ определяется как $d_{Q}=d_{Q}^{k}+2 d_{Q}^{\omega}$ и играет ту же роль в теории перенормировки нашей динамической модели, что и простая импульсная размерность в статических моделях.

Подробный размерностный анализ действия (5) для пространства размерности $d>2$ [7], [13] показывает, что модель является логарифмической при $\varepsilon=0$, т. е. постоянная взаимодействия $g_{0}$ безразмерна в этой точке, и что кажущиеся УФ-расходимости имеют вид полюсов по $\varepsilon$ и представлены только для 1-неприводимых функций $\left\langle v_{i}^{\prime} v_{j}\right\rangle_{1-i r}$ и $\left\langle\theta^{\prime} \theta\right\rangle_{1-i r}$ со следующими контрчленами: $v_{i}^{\prime} \Delta v_{j}$ и $\theta^{\prime} \Delta \theta$. Их 
можно явно выразить через мультипликативную перенормировку затравочных параметров $g_{0}, \nu_{0}$ и $u_{0}$ без перенормировок полей:

$$
\nu_{0}=\nu Z_{\nu}, \quad g_{0}=g \mu^{2 \varepsilon} Z_{g}, \quad u_{0}=u Z_{u},
$$

где безразмерные параметры $g, \nu$ и $u$ представляют собой перенормированные аналоги соответствующих затравочных параметров, $\mu$ - перенормированная масса (масштабообразующий параметр), артефакт схемы регуляризации, а $Z_{i}=Z_{i}(g, u, \rho, d, \varepsilon)-$ так называемые константы перенормировки, $i=g, \nu, u$.

Таким образом, перенормированный функционал действия имеет вид

$$
\begin{aligned}
S^{\mathrm{R}}(\Phi)=\frac{1}{2} & \int d t_{1} d^{d} \mathbf{x}_{1} d t_{2} d^{d} \mathbf{x}_{2}\left[v_{i}^{\prime}\left(t_{1}, \mathbf{x}_{1}\right) D_{i j}^{v}\left(t_{1}, \mathbf{x}_{1} ; t_{2}, \mathbf{x}_{2}\right) v_{j}^{\prime}\left(t_{2}, \mathbf{x}_{2}\right)+\right. \\
& \left.+\theta^{\prime}\left(t_{1}, \mathbf{x}_{1}\right) D^{\theta}\left(t_{1}, \mathbf{x}_{1} ; t_{2}, \mathbf{x}_{2}\right) \theta^{\prime}\left(t_{2}, \mathbf{x}_{2}\right)\right]+ \\
& +\int d t d^{d} \mathbf{x}\left\{\theta^{\prime}\left[-\partial_{t}-\mathbf{v} \cdot \boldsymbol{\partial}+\nu u Z_{2} \Delta\right] \theta+\mathbf{v}^{\prime}\left[-\partial_{t}-\mathbf{v} \cdot \boldsymbol{\partial}+\nu Z_{1} \Delta\right] \mathbf{v}\right\}
\end{aligned}
$$

Сравнивая перенормированное действие (6) с определениями констант пренормировки $Z_{i}, i=g, \nu, u$, и учитывая, что $g_{0} \nu_{0}^{3}=g \nu^{3} \mu^{2 \varepsilon}$ [13], получаем соотношения между $Z_{g}, Z_{\nu}, Z_{u}$ и $Z_{1}, Z_{2}$, а именно

$$
Z_{g}=Z_{1}^{-3}, \quad Z_{\nu}=Z_{1}, \quad Z_{u}=Z_{2} Z_{1}^{-1}
$$

Это означает, что остаются две независимые константы перенормировки $Z_{1}$ и $Z_{2}$, и в схеме минимальных вычитаний (MB), которую мы будем в дальнейшем использовать, они имеют следующий явный вид:

$$
\begin{gathered}
Z_{1}(g, \rho, d, \varepsilon)=1+\sum_{n=1}^{\infty} g^{n} \sum_{j=1}^{n} \frac{z_{n j}^{(1)}(\rho, d)}{\varepsilon^{j}}, \\
Z_{2}(g, \rho, u, d, \varepsilon)=1+\sum_{n=1}^{\infty} g^{n} \sum_{j=1}^{n} \frac{z_{n j}^{(2)}(\rho, u, d)}{\varepsilon^{j}}
\end{gathered}
$$

где коэффициенты $z_{n j}^{(i)}, i=1,2$, не зависят от $\varepsilon$. Их явный вид можно определить, потребовав, чтобы 1 -неприводимые функции Грина $\left\langle v_{i}^{\prime} v_{j}\right\rangle_{1-i r}$ и $\left\langle\theta^{\prime} \theta\right\rangle_{1-i r}$, записанные через перенормированные переменные, были УФ-конечными, т. е. они не должны содержать полюсов по $\varepsilon$. С другой стороны, 1-неприводимые функции Грина $\left\langle v_{i}^{\prime} v_{j}\right\rangle_{1-i r}$ и $\left\langle\theta^{\prime} \theta\right\rangle_{1-i r}$ связаны с операторами собственной энергии $\Sigma^{v^{\prime} v}$ и $\Sigma^{\theta^{\prime} \theta}$ уравнениями Дайсона, которые можно записать в следующем удобном виде:

$$
\begin{aligned}
\left\langle v_{i}^{\prime} v_{j}\right\rangle_{1-i r} & =\left(-i \omega+\nu_{0} p^{2}\right) P_{i j}-\Sigma_{i j}^{v^{\prime} v}(\omega, p), \\
\left\langle\theta^{\prime} \theta\right\rangle_{1-i r} & =-i \omega+\nu_{0} u_{0} p^{2}-\Sigma^{\theta^{\prime} \theta}(\omega, p) .
\end{aligned}
$$

Таким образом, коэффициенты $z_{n j}^{(i)}$ констант перенормировки $Z_{i}, i=1,2$, находятся из требования, чтобы УФ-расходимости сокращались в (10) и (11) после подстановок $\nu_{0}=\nu Z_{1}$ в $(10)$ и $\nu_{0} u_{0}=\nu u Z_{2}$ в (11). Это определяет $Z_{i}, i=1,2$, с точностью до конечного УФ-вклада, который фиксируется выбором схемы перенормировки. 
Как уже отмечалось, мы работаем в рамках схемы МВ со стандартным видом констант $Z_{i}, i=1,2$, приведенным в (8) и (9).

В неспиральном случае $(\rho=0)$ разложение константы перенормировки $Z_{i}$ известно с точностью до второго порядка по $g$ (двухпетлевое приближение), явный вид коэффициентов $z_{11}^{(i)}, z_{21}^{(i)}$ и $z_{22}^{(i)}, i=1,2$, был получен в работах [7], [8], [13]. Простейший однопетлевой результат для $z_{11}^{(i)}$ таков:

$$
z_{11}^{(1)}=-\frac{S_{d}}{(2 \pi)^{d}} \frac{(d-1)}{8(d+2)}, \quad z_{11}^{(2)}=-\frac{S_{d}}{(2 \pi)^{d}} \frac{(d-1)}{4 d u(1+u)},
$$

где $S_{d} \equiv 2 \pi^{d / 2} / \Gamma(d / 2)$ - площадь поверхности $d$-мерной единичной сферы, а $\Gamma(x)-$ гамма-функция Эйлера. Неспиральные двухпетлевые поправки $z_{21}^{(i)}$ представляют собой довольно громоздкие выражения, которые можно найти в работах [7], [8], [13]. С другой стороны, коэффициенты $z_{22}^{(i)}$ просто связаны с однопетлевыми коэффициентами $z_{11}^{(i)}$, однако в дальнейшем они нам не понадобятся. Кроме того, явная зависимость коэффициента $z_{21}^{(1)}$ от параметра спиральности также известна [14], поэтому для анализа роли спиральности в предлагаемой модели (в рамках двухпетлевого приближения) необходимо найти явную зависимость коэффициента $z_{21}^{(2)}$ от параметра спиральности. Чтобы найти эту зависимость, требуется вычислить восемь двухпетлевых фейнмановских диаграмм (диаграмм собственной энергии для оператора собственной энергии $\Sigma^{\theta^{\prime} \theta}$, определяемых из (11)), которые представлены на рис. 3 (подробности см. в работе [7]). В общем случае выражения получаются довольно громоздкими, поэтому в настоящей работе мы не приводим все результаты. Для наших целей достаточно представить окончательное численное выражение для коэффициента $z_{21}^{(2)}$ как функции от параметра спиральности $\rho$ для пространственной размерности $d=3$ (единственной пространственной размерности, существенной для спирального случая):

$$
z_{21}^{(2)}=-1.1374 \cdot 10^{-5}+0.9812 \cdot 10^{-5} \rho^{2},
$$

где уже использовано однопетлевое значение неподвижной точки для параметра $u_{*}^{(1)}=1.393$ (см. ниже).

Прежде чем начать исследование турбулентного числа Прандтля в спиральной среде, кратко обсудим устойчивость соответствующего скейлингового режима.

Тот факт, что все поля модели не перенормируются, означает, что перенормированные корреляционные функции $W^{\mathrm{R}}=\langle\Phi \ldots \Phi\rangle^{\mathrm{R}}$ равны своим неперенормированным аналогам $W=\langle\Phi \ldots \Phi\rangle$. Единственное различие коренится в выборе переменных (перенормированных или неперенормированных) и в соответствующем пертурбативном разложении (по $g$ или $g_{0}$ ),

$$
W^{\mathrm{R}}(g, \nu, u, \mu, \ldots)=W\left(g_{0}, \nu_{0}, u_{0}, \ldots\right),
$$

где точки означают остальные аргументы, которые не затрагиваются перенормировкой, например параметр спиральности, координаты, времена и т. д. Используя тот факт, что неперенормированные корреляционные функции не зависят от масштабообразующего параметра $\mu$, можно применить дифференциальный оператор 

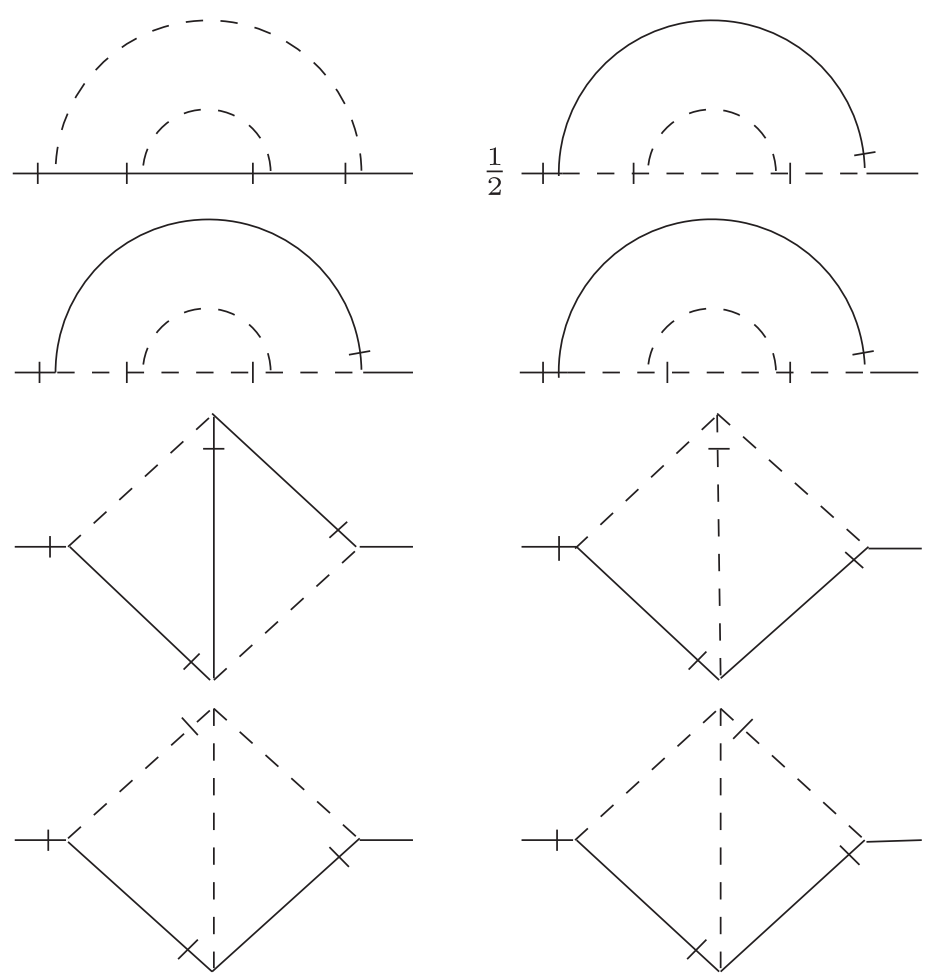

Рис. 3. Двухпетлевые диаграммы собственной энергии для оператора собственной энерги $\Sigma^{\theta^{\prime} \theta}$. Соответствующие пропагаторы и вершины показаны соответственно на рис. 1 и 2.

$\mathcal{D}_{\mu} \equiv \mu \partial_{\mu}$ при фиксированных затравочных (неперенормированных) параметрах к обеим сторонам равенства (13), что приводит к основному дифференциальному уравнению РГ:

$$
\left[\mu \partial_{\mu}+\beta_{g}(g) \partial_{g}+\beta_{u}(g, u) \partial_{u}-\gamma_{\nu}(g) \nu \partial_{\nu}\right] W^{\mathrm{R}}(g, \nu, u, \mu, \ldots)=0
$$

где так называемые РГ-функции ( $\beta$ - и $\gamma$-функции) задаются как

$$
\begin{gathered}
\beta_{g} \equiv \mu \partial_{\mu} g=g\left(-2 \varepsilon+3 \gamma_{1}\right), \quad \beta_{u} \equiv \mu \partial_{\mu} u=u\left(\gamma_{2}-\gamma_{1}\right), \\
\gamma_{i} \equiv \mu \partial_{\mu} \ln Z_{i}, \quad i=1,2,
\end{gathered}
$$

и мы воспользовались соотношениями между константами перенормировки, заданными в (7).

Хорошо известно, что инфракрасное асимптотическое скейлинговое ИК-поведение (скейлинговое поведение в рамках инерциального диапазона) корреляционных функций соответствующей модели управляется ИК-устойчивой неподвижной точкой уравнений РГ. Неподвижная точка получается из требования обращения в ноль функций $\beta_{g}$ и $\beta_{u}$ (в этом случае уравнения РГ (14) становятся дифференциальными уравнениями для обобщенно-однородных функций $W^{\mathrm{R}}$, т. е. их решения имеют 
скейлинговый вид). Таким образом, в нашем случае координаты неподвижной точки даются уравнениями

$$
\beta_{g}\left(g_{*}\right)=g_{*}\left(-2 \varepsilon+3 \gamma_{1}^{*}\right)=0, \quad \beta_{u}\left(g_{*}, u_{*}\right)=u_{*}\left(\gamma_{2}^{*}-\gamma_{1}^{*}\right)=0,
$$

где значения всех величин в неподвижной точке обозначены звездочкой. Отсюда немедленно получаем точные значения $\gamma_{i}^{*}=2 \varepsilon / 3, i=1,2$, аномальной размерности в неподвижной точке $g_{*} \neq 0, u_{*} \neq 0$. Вид функций $\beta_{g}$ и $\beta_{u}$ в $(15)$ не зависит от порядка разложения по теории возмущений, т. е. он точно задается уже в однопетлевом приближении в рамках теории возмущений без высших петлевых поправок.

Тем не менее для определения ИК-притяжения соответствующей неподвижной точки необходим явный вид констант перенормировки $Z_{i}, i=1,2$, который определяет явный вид аномальных размерностей $\gamma_{i}(15)$. Достаточным условием для ИК-устойчивости неподвижной точки является положительность вещественных частей собственных значений матрицы первых производных

$$
\Omega_{k l}^{*}=\left(\frac{\partial \beta_{k}}{\partial l}\right)_{*}, \quad k, l=g, u,
$$

взятых в неподвижной точке $\left(g_{*}, u_{*}\right)$. Здесь требуется явный вид неподвижной точки. В спиральном случае при точном значении $d=3$ координаты неподвижной точки имеют вид

$$
g_{*}=\frac{40 \pi^{2}}{3} \varepsilon\left(1+\left(-1.101+0.743 \rho^{2}\right) \varepsilon\right), \quad u_{*}=1.393+\left(0.0712-0.2436 \rho^{2}\right) \varepsilon,
$$

в котором выделена явная зависимость от параметра спиральности $\rho$.

Теперь, используя значения неподвижных точек (17) в матрице первых производных (16), можно прямыми вычислениями показать, что оба собственных значения имеют положительную вещественную часть при всех допустимых значениях $|\rho|<1$ параметра спиральности $\rho$. Это означает, что устойчивость соответствующего колмогоровского скейлингового ИК-режима не нарушается в результате наличия спиральности, хотя соответствующее собственное значение убывает при возрастании абсолютного значения параметра спиральности.

\section{5. СПИРАЛЬНОСТЬ И ТУРБУЛЕНТНОЕ ЧИСЛО ПРАНДТЛЯ}

Теперь у нас имеются все необходимые результаты для анализа влияния спиральности на турбулентное число Прандтля. В работе [7] была получена формула для точного двухпетлевого приближения обратного турбулентного (эффективного) числа Прандтля $u_{\mathrm{eff}}$ :

$$
\begin{aligned}
u_{\mathrm{eff}}=u_{*}^{(1)} & \left(1+\varepsilon\left\{\frac{1+u_{*}^{(1)}}{1+2 u_{*}^{(1)}}\left[\lambda-\frac{128(d+2)^{2}}{3(d-1)^{2}} B\left(u_{*}^{(1)}\right)\right]+\right.\right. \\
+ & \left.\left.\frac{(2 \pi)^{d}}{S_{d}} \frac{8(d+2)}{3(d-1)}\left(a_{v}-a_{\theta}\right)\right\}\right) .
\end{aligned}
$$

Величины в данном уравнении имеют следующий смысл: $u_{*}^{(1)}$ - однопетлевое значение параметра $u$ в неподвижной точке (оно также является однопетлевым значением 


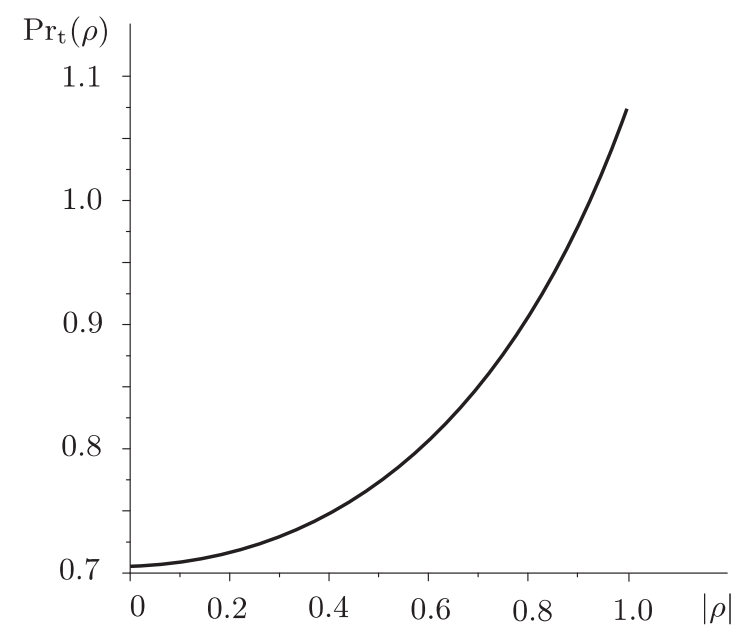

Рис. 4. Зависимость турбулентного числа Прандтля от абсолютного значения параметра спиральности $\rho$.

для обратного турбулентного числа Прандтля). Для общего случая $d>2$ оно задается следующим простым уравнением [7]:

$$
u_{*}^{(1)}\left[1+u_{*}^{(1)}\right]=2 \frac{d+2}{d} .
$$

При $d=3$ получаем $u_{*}^{(1)}=1.393$. Значение $\lambda$ связано с коэффициентом $z_{21}^{(1)}$ в $(8)$, т. е. оно получается с помощью двухпетлевого ренормгруппового анализа модели чистой полностью развитой турбулентности на основании стохастического уравнения Навье-Стокса. В работе [13] это значение было получено для неспирального случая, а в работе [14] была вычислена его зависимость от параметра спиральности. При $d=3$ получаем $\lambda(\rho)=-1.101+0.743 \rho^{2}$. Кроме того, в (18) величины $a_{v}$ и $a_{\theta}$ даются соответствующими разложениями в главном порядке по $\varepsilon$ скейлинговых функций: функций отклика $\left\langle v v^{\prime}\right\rangle$ и $\left\langle\theta \theta^{\prime}\right\rangle$ для поля скоростей и скалярного поля соответственно (подробности см. в работе [7]). Их значения для $d=3$ (в спиральном и неспиральном случаях) таковы:

$$
a_{v}=-\frac{0.047718}{2 \pi^{2}}, \quad a_{\theta}=-\frac{0.04139}{2 \pi^{2}} .
$$

Наконец, величина $B\left(u_{*}^{(1)}\right)$ в формуле $(18)$ при $d=3$ (спиральный случай) связана с коэффициентом $z_{21}^{(2)}$, заданным равенством $(12)$, простым соотношением (подробности см. в работах [7], [8])

$$
B\left(u_{*}^{(1)}, \rho\right)=\frac{(2 \pi)^{6}}{S_{3}^{2}} z_{21}^{(2)}=-4.432 \cdot 10^{-3}+3.823 \cdot 10^{-3} \rho^{2} .
$$

Таким образом, используя все определенные выше величины, в случае $d=3$ мы приходим к следующему окончательному двухпетлевому выражению для обратного турбулентного числа Прандтля в спиральной турбулентной среде:

$$
u_{\mathrm{eff}}=1.393+\left(0.012444-0.243352 \rho^{2}\right) \varepsilon+O\left(\varepsilon^{2}\right),
$$


и для физического значения $\varepsilon=2$ окончательно получаем

$$
\operatorname{Pr}_{\mathrm{t}}^{-1}=u_{\text {eff }}=1.4179-0.4867 \rho^{2} .
$$

Зависимость турбулентного числа Прандтля от параметра спиральности показана на рис. 4. Как можно видеть, наличие спиральности увеличивает значение турбулентного числа Прандтля, и в случае полностью спиральной турбулентной системы при $|\rho|=1$ вклад спиральности в турбулентное число Прандтля составляет почти $50 \%$ от неспирального вклада. Из рис. 4 можно увидеть интересный факт: наличие спиральности в предложенной модели сдвигает значение турбулентного числа Прандтля вглубь экспериментально полученного интервала $[0.7,0.9][1],[2]$. В связи с этим немедленно возникает вопрос о влиянии, которое оказывает нарушение симметрии на экспериментально полученные значения турбулентного числа Прандтля.

\section{6. ЗАКЛЮЧЕНИЕ}

В настоящей работе с использованием теоретико-полевого подхода РГ в рамках двухпетлевого приближения проанализировано влияние наличия спиральности (нарушения пространственной четности) в полностью развитых турбулентных потоках на процессы диффузии пассивно увлекаемой скалярной величины. В первую очередь показано, что наличие спиральности не приводит к нарушению устойчивости соответствующевого скейлингового режима. Более того, турбулентное число Прандтля найдено как функция параметра спиральности. Показано, что спиральность приводит к увеличению турбулентного числа Прандтля вплоть до 50\%-го превышения над неспиральным случаем. Полученные результаты демонстрируют, что отклонения от полностью симметричных турбулентных систем могут иметь нетривиальные последствия для универсальных характеристик турбулентных сред.

Благодарности. Авторы благодарят за гостеприимство Лабораторию теоретической физики им. Н.Н. Боголюбова в Объединенном институте ядерных исследований (Дубна, Россия). Настоящая работа была поддержана Slovak Academy of Sciences (грант VEGA № 2/0173/09) и проектом ITMS № 26220120029 (supporting operational Research and development program, European Regional Development Fund).

\section{Список литературы}

[1] А.С. Монин, А.Н. Яглом, Статистическая гидромеханика, т. 1, Наука, М., 1965.

[2] L.P. Chua, R. A. Antonia, Internat. J. Heat Mass Transfer, 33:2 (1990), 331-339; K. Chang, E. A. Cowen, J. Eng. Mech., 128:10 (2002), 1082, 6 pp.

[3] W. D. McComb, The Physics of Fluid Turbulence, Oxford Engineering Sci. Ser., 25, Oxford Univ. Press, Oxford, 1990.

[4] Л. Ц. Аджемян, Н. В. Антонов, А. Н. Васильев, УФН, 166:12 (1996), 1257-1284.

[5] L. Ts. Adzhemyan, N. V. Antonov, A. N. Vasil'ev, The Field Theoretic Renormalization Group in Fully Developed Turbulence, Gordon and Breach, London, 1999.

[6] А.Н. Васильев, Квантовополевая ренормгруппа в теории критического поведения и стохастической динамике, Изд-во ПИЯФ, СПб., 1998.

[7] L. Ts. Adzhemyan, J. Honkonen, T. L. Kim, L. Sladkoff, Phys. Rev. E, 71:5 (2005), 056311, 9 pp., arXiv: physics/0412019.

[8] E. Jurčišinová, M. Jurčišin, R. Remecký, Phys. Rev. E, 82:2 (2010), 028301, 3 pp. 
[9] Л. Ц. Аджемян, А. Н. Васильев, М. Гнатич, ТМФ, 58:1 (1984), 72-78.

[10] G. Falkovich, K. Gawȩdzki, M. Vergassola, Rev. Modern. Phys., 73 (2001), 931-975; L. Biferale, I. Procaccia, Phys. Rep., 414:2-3 (2005), 43-164, arXiv: nlin/0404014; N. V. Antonov, J. Phys. A, 39:25 (2006), 7825-7865.

[11] O. G. Chkhetiani, M. Hnatich, E. Jurčišinová, M. Jurčišin, A. Mazzino, M. Repašan, Phys. Rev. E, 74:3 (2006), 036310, 26 pp., arXiv: nlin/0603030.

[12] P. C. Martin, E. D. Siggia, H. A. Rose, Phys. Rev. A, 8:1 (1973), 423-437; C. De Dominicis, J. Phys. Colloques, 37:C1 (1976), 247-254; H.-K. Janssen, Z. Phys. B, 23:4 (1976), 377-380; R. Bausch, H. K. Janssen, H. Wagner, Z. Phys. B, 24:1 (1976), 113-127.

[13] L. Ts. Adzhemyan, N. V. Antonov, M. V. Kompaniets, A. N. Vasil'ev, Internat. J. Modern Phys. B, 17:10 (2003), 2137-2170, arXiv: nlin/0207007.

[14] E. Jurčišinová, M. Jurčišin, R. Remecký, Phys. Rev. E, 79:4 (2009), 046319, 10 pp. 